\title{
Enabling large scale capacitive sensing for dielectric elastomers
}

\author{
Daniel Xü ${ }^{* a}$, Thomas G. McKay ${ }^{\mathrm{a}}$, Silvain Michel ${ }^{\mathrm{b}}$, Iain A. Anderson ${ }^{\mathrm{a}, \mathrm{c}}$ \\ ${ }^{a}$ Biomimetics Lab, Auckland Bioengineering Institute, University of Auckland, 70 Symonds Street, \\ Auckland, New Zealand \\ ${ }^{b}$ Mechanical Systems Engineering Lab, Empa Materials Science Technology, Ueberlandstrasse 129, \\ CH-8600 Dübendorf, Switzerland \\ ${ }^{c}$ Engineering Science, University of Auckland, 70 Symonds Street, Auckland, New Zealand
}

\begin{abstract}
Hand motion is one of our most expressive abilities. By measuring our interactions with everyday objects, we can create smarter artificial intelligence that can learn and adapt from our behaviours and patterns. One way to achieve this is to apply wearable dielectric elastomer strain sensors directly onto the hand.

Applications such as this require fast, efficient and scalable sensing electronics. Most capacitive sensing methods use an analogue sensing signal and a backend processor to calculate capacitance. This not only reduces scalability and speed of feedback but also increases the complexity of the sensing circuitry.
\end{abstract}

A capacitive sensing method that uses a DC sensing signal and continuous tracking of charge is presented. The method is simple and efficient, allowing large numbers of dielectric elastomer sensors to be measured simulatenously.

Keywords: Dielectric elastomer sensor, capacitive sensing, charge tracking, DC input voltage

\section{INTRODUCTION}

Artificial intelligence (AI) in computers and personal devices has dramatically simplified our lives. Now in our pockets, homes and even as medical implants, we are giving AI more control and trust to assist us in our daily activities. At the front-end of $\mathrm{AI}$ is a set of sensor inputs that gather information to perform their specified tasks such as changing the temperature on the thermostat, shuffling the songs on a music player and even reminding us to take our medication; tasks that require the knowledge of our mood, behaviour and state.

To train smarter AI that understand our behaviour, we need sensors to measure our behaviour. This allows AI to learn from our movement patterns and adapt to our needs. Already, we are using wearable technologies such as the Fitbit ${ }^{\circ}$ to monitor our physical activity and quality of sleep. But existing wearable technologies lack the ability to measure hand gestures and movement.

Much of our interaction with the world is through our hands. Think of the number of items you touch over the course of a day. We not only rely on them to function, but also to convey ideas and feelings. In a way, they are an extension of our mind and personality. There is a rich set of information that can be extracted by studying the interactions and patterns of our hands ${ }^{1}$. For instance, by detecting tension in the grip on a steering wheel, AI can be programmed to play soothing music to calm the driver of a car. By measuring these interactions, AI can learn more about our behaviours and personalities making them even smarter.

However, accurately measuring hand motion is particularly difficult given its 27 degrees of freedom ${ }^{2}$ and a high level of obstruction. One unobtrusive way to achieve this is through soft wearable sensors. Dielectric elastomer (DE) artificial muscles are electroactive polymers with muscle like capabilities ${ }^{3,4}$. Like real muscles, they can sense their state when strained thus giving them motion sensing abilities. To effectively measure hand movement to a high degree of resolution,

* Author to whom correspondence should be addressed: dxu011@aucklanduni.ac.nz

Electroactive Polymer Actuators and Devices (EAPAD) 2014, edited by Yoseph Bar-Cohen, Proc. of SPIE Vol. 9056, 90561A · C 2014 SPIE · CCC code: 0277-786X/14/\$18 · doi: 10.1117/12.2044356 
we require fast, efficient and scalable methods. While it is relatively simple to measure the state of one sensor, the challenge comes with scaling. Due to technical limitations, existing sensing methods are ill-equipped to handle large numbers of sensors. This paper first reviews some common sensing methods, then presents a fast, efficient and scalable solution for multi-sensing systems.

\subsection{Artificial muscle sensors}

DE's are constructed by sandwiching a soft dielectric material between compliant electrodes (Figure 1, left), resembling a flexible capacitor. When used as a strain sensor, they are typically operated under low voltages which simplifies their electrical model to a variable resistor $\left(R_{S}\right)$ in series with a variable capacitor $(C)^{3,4}$ (Figure 1, right).

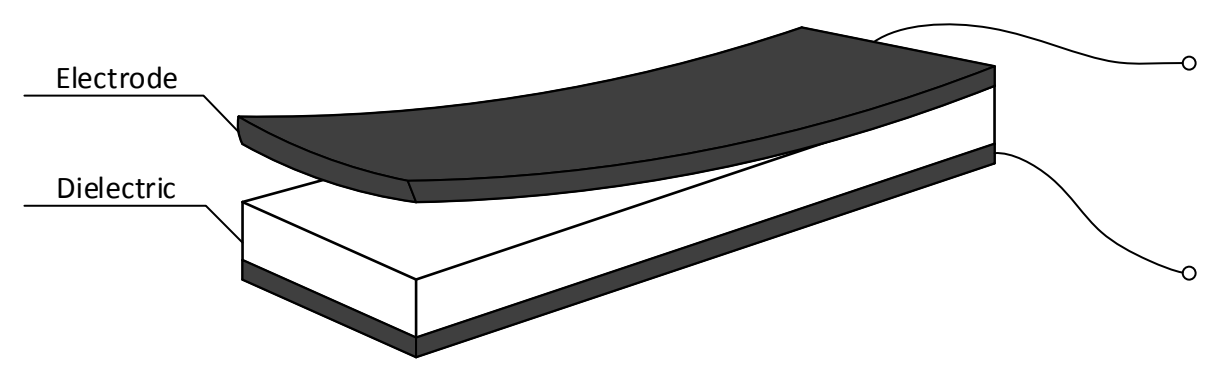

Electrical model

Figure 1. Dielectric elastomer construction (left) and its equivalent electrical model (right).

Although resistance can be used to infer strain, results are highly dependent on temperature and electrode quality ${ }^{7}$ A more accurate alternative is by measuring capacitance ${ }^{5,8-10}$, a property dependent on the overlapping area of the electrodes $(A)$, thickness of the dielectric $(d)$, relative permittivity of the dielectric $\left(\varepsilon_{r}\right)$ and the permittivity of free space $\left(\varepsilon_{0}\right)(1)$

$$
C=\frac{\varepsilon_{r} \varepsilon_{0} A}{d}
$$

Unfortunately, measuring the capacitance of DEs is not a trivial task. Typically, the electrodes are made with particles that are carbon based to maintain conductivity at large strains. Their resistance can be up to 100 's of $\mathrm{k} \Omega \mathrm{s}^{11}$ and is also strain dependent. Most capacitance meters were not designed to handle any significant electrode resistance and therefore unable to correctly measure the DE's capacitance ${ }^{12}$.

\section{CAPACITIVE SENSING METHODS FOR DIELECTRIC ELASTOMERS}

To accurately measure the DE's capacitance, its electrode resistance also needs to be taken into account. This is challenging because the electrode resistance causes an internal voltage drop, which affects the voltage on the capacitor. Common sensing methods that account for this include measuring the gain and phase shift of current from a sinusoidal voltage input ${ }^{5}$, the impedance frequency response ${ }^{8}$, a linear regression on the DE's voltage and current output from a period of arbitrary excitation ${ }^{10}$ and integrating the charging current over half the period of a switching waveform ${ }^{13}$.

\subsection{Capacitance from gain and phase shift of a sinusoidal input}

This method ${ }^{5}{ }^{6}$ requires an accurate measurement of the gain and phase shift of current $(I)$ from a sinusoidal input voltage $(V)$. Given a voltage input of $A \cos (\omega t)$, current can be expressed as $B \cos (\omega t+\varphi)$, where $A$ and $B$ are their respective amplitudes and $\varphi$ is the phase shift (Figure 2). 


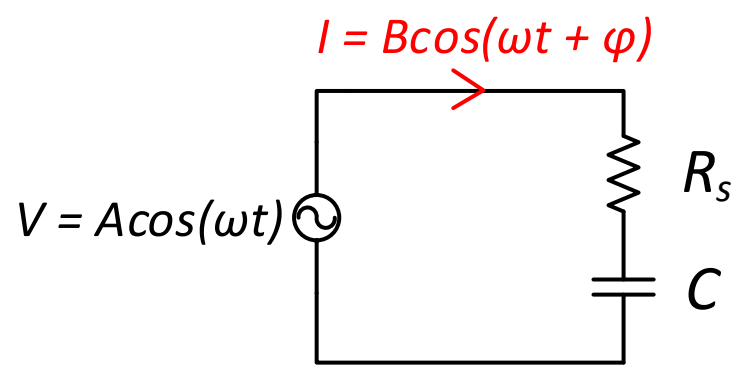

Figure 2. The current through the DE from a sinusoidal voltage input is of the same frequency with a gain and phase shift.

For a relatively fast sensing frequency $(\omega)$, the DE's geometry can be assumed constant during the entire period of the sensing signal. This allows capacitance to be calculated by simultaneously solving the magnitude and phase equations (2) $\&$ (3) respectively.

$$
\begin{aligned}
& \frac{B}{A}=\frac{\omega C}{\sqrt{1+\left(\omega R_{S} C\right)^{2}}} \\
& \varphi=\tan ^{-1}\left(\frac{1}{\omega R_{S} C}\right)
\end{aligned}
$$

\subsection{Capacitance from impedance frequency response}

This method ${ }^{8}$ requires the measurement of impedance at multiple sensing frequencies. The complex impedance of the DE $\left(Z_{T}\right)$ is the sum of the impedance of the electrode resistance $\left(R_{S}\right)$ and the impedance of the capacitor $\left(\frac{1}{j \omega C}\right)(4)$.

$$
Z_{T}=R_{s}+\frac{1}{j \omega C}
$$

Because the impedance of the capacitor decreases with frequency, the total impedance $\left(Z_{T}\right)$ reduces to only the electrode resistance $\left(R_{S}\right)$ at high frequencies (Figure 3). At low frequencies, the system behaves similarly to a high pass filter ${ }^{8,9}$ and the $3 \mathrm{~dB}$ attenuation cut-off frequency $\left(f_{c}\right)$ can be used to calculate capacitance $(5)$.

$$
f_{c}=\frac{1}{2 \pi R_{s} C}
$$

\section{DE's impedance frequency response}

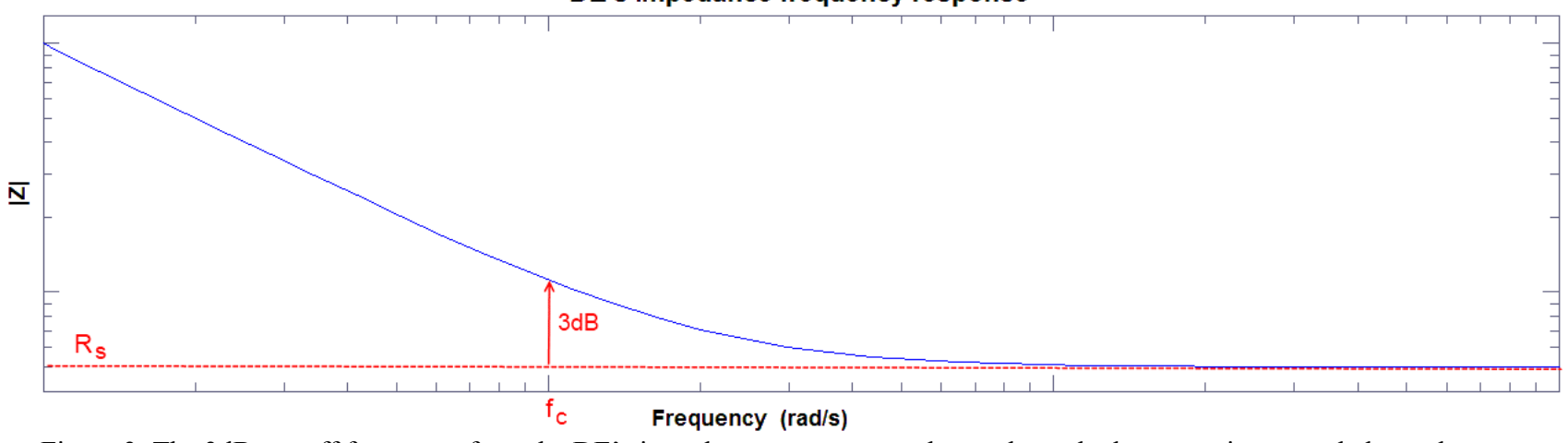

Figure 3. The $3 \mathrm{~dB}$ cut-off frequency from the DE's impedance response can be used to calculate capacitance and electrode resistance. 


\subsection{Capacitance from Hyper-plane approximation}

One method that allows an arbitrary sensing signal to be used is the Hyper-plane approximation ${ }^{10}$. This method performs a linear regression on the sensor's voltage $(V)$, current $(I)$ and charge $(Q)$ over a period of sensing input. Capacitance is represented as the gradient $\frac{\partial Q}{\partial V}$ of the data collected. Graphically, this corresponds to the plane of best fit in the $Q V$ plane (Figure 4). A drawback to this method is that the process is computationally intensive and usually requires a backend processor to post-process the data.

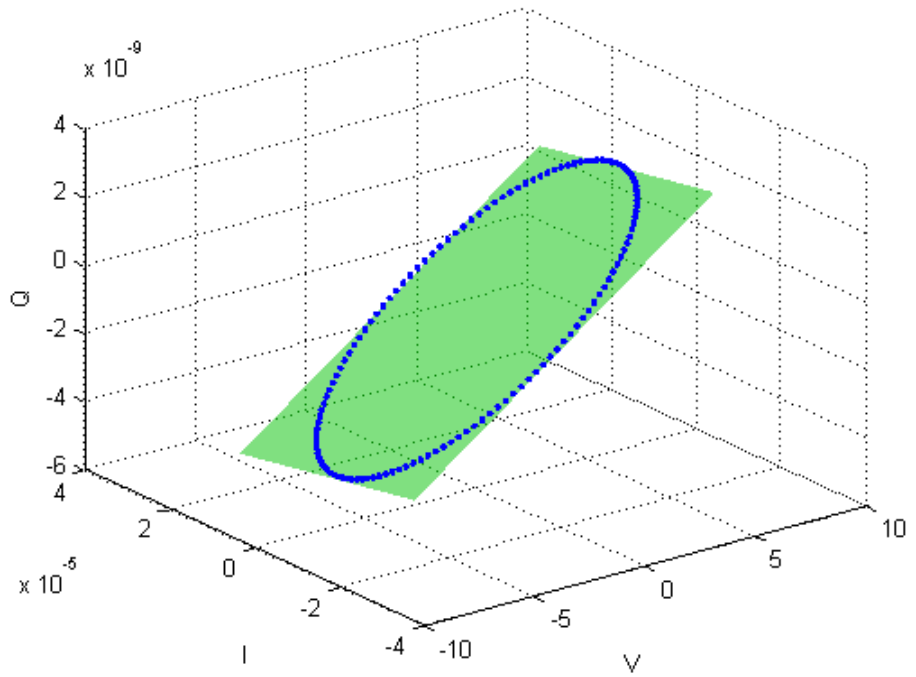

Figure 4. The Hyper-plane approximation method to measure capacitance is calculated from the plane of best fit of the DE's $V, I$ and $Q$ in the $Q V$ plane (green).

\subsection{Capacitance from current integration}

This method ${ }^{13}$ uses the governing capacitor voltage equation, defined as the ratio of charge $(Q)$ to voltage $\left(V_{c}\right)(6)$.

$$
C=\frac{Q}{V_{c}}
$$

For a step response, the capacitor voltage $\left(V_{c}\right)$ approaches the supply voltage $\left(V_{s}\right)$ after approximated $3 R C$ time constants (Figure 5).

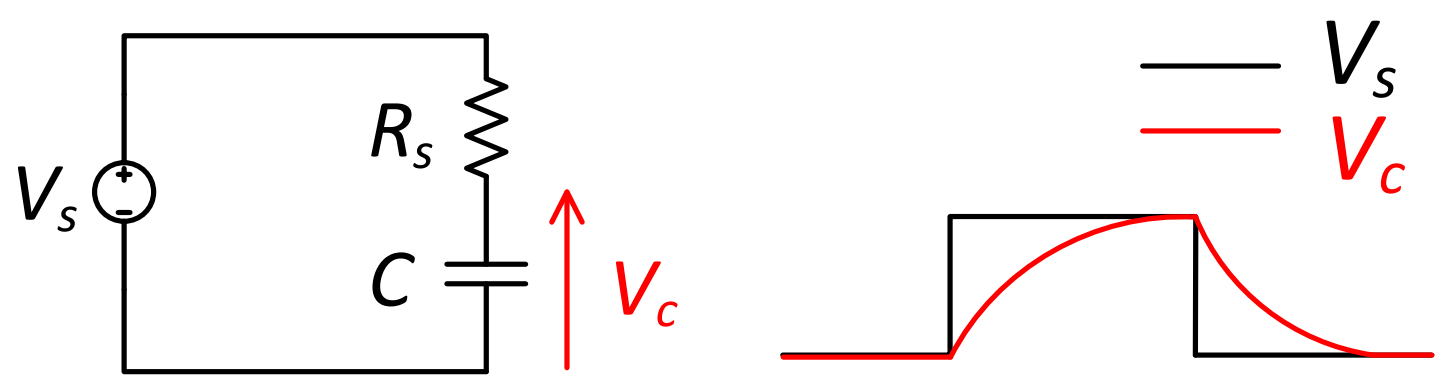

Figure 5. After approximately $3 \mathrm{RC}$ time constants, $V_{c}$ equals the same value as $V_{s}$.

By using a square wave sensing signal with a sufficiently long period and integrating current $(I)$ to measure charge $(Q)$, capacitance can be evaluated once the capacitor voltage $\left(V_{c}\right)$ equals the supply voltage $\left(V_{s}\right)(7)$. Capacitance is calculated after each half period of the sensing signal.

$$
C=\frac{\int I d t}{V_{S}}
$$




\section{CONTINUOUS CHARGE TRACKING TO MEASURE CAPACITANCE}

To effectively use DEs to measure hand motion to a high degree of resolution, the sensing method needs to be fast, efficient and scalable. With the exception of current integration ${ }^{13}$, all other methods require a backend processor to perform feature detection or complex algorithms to calculate capacitance. Each additional sensing channel will increase the computation load and therefore reduce the rate of capacitive feedback.

Current integration ${ }^{13}$ is simple but it is inefficient because the DE is repeatedly charged and then discharged. This causes unnecessary losses through the electrode resistance and requires waiting for the capacitor to become fully charged before capacitance can be measured.

A modification of this method replaces the square wave sensing voltage with a constant DC voltage and continuously tracks the movement of charge to and from the DE. Recall that capacitance is the ratio of charge to voltage at any instant in time (6). Hence under a constant DC voltage, changes to capacitance (as a result of strain) is proportionally reflected as a movement of charge. Stretching the DE increases its capacitance, drawing more charge onto the DE. While relaxing the DE reduces its capacitance, removing change from the DE (Figure 6). Current is continuously integrated to determine total charge on the DE.

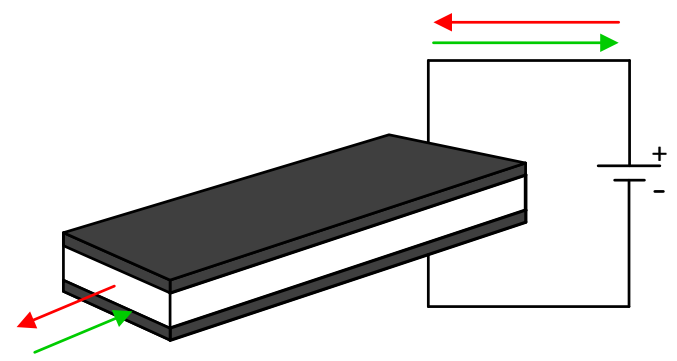

Figure 6. Under a constant voltage, stretching adds charge to the DE (red) while relaxing removes charge from the DE (green).

Using a constant DC voltage simplifies the sensing electronics and allows the DE's capacitance to dictate the movement of charge (Figure 7). This prevents any unnecessary discharge, thus results in shorter transient times compared to recharging completely from zero. For a typical sensor designed to measure hand motion, the transient charge time is usually much faster than the speed of muscle contraction ${ }^{* *}$. Hence the DE is constantly in steady state (constant charge) and (7) can be used to calculate capacitance.

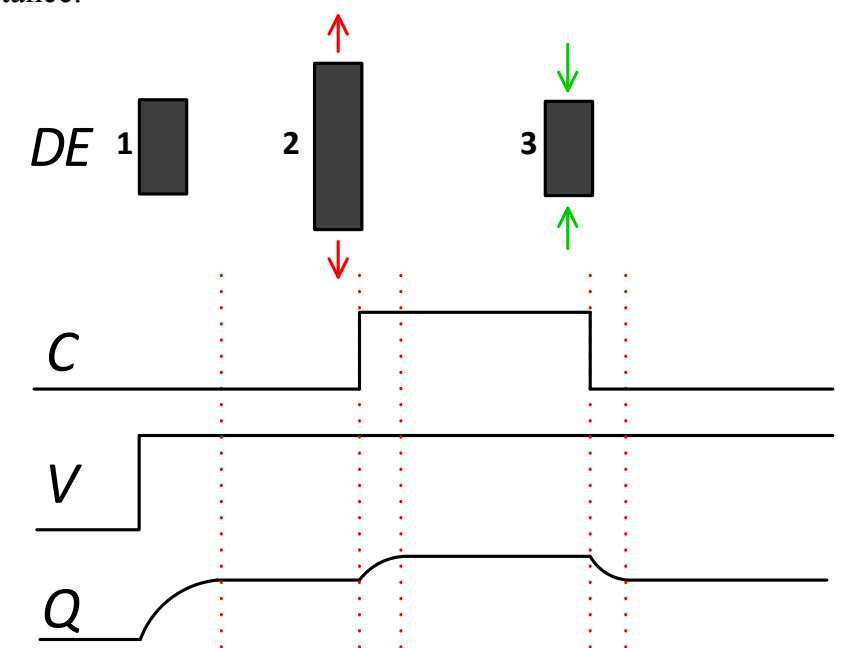

Figure 7. Capacitance, voltage and charge profiles. (1) The initial turn on causes the capacitor to charge to the value $Q=C V$. Once the system reaches steady state, charge remains constant. (2) Increasing the capacitance adds more charge to the DE. (3) Decreasing the capacitance reduces charge on the DE.

** A typical $10 \mathrm{~cm} \times 10 \mathrm{~cm}$ sensor is likely to have a transient period under $1 \mathrm{~ms}$. 
To account for any integration drift, a switching component can be used to periodically disconnect the DC voltage and reset the integrator. This can be either performed manually or programmed with a hardware timer. A complete analogue implementation of charge tracking to measure the DE's capacitance is shown in Figure 8.

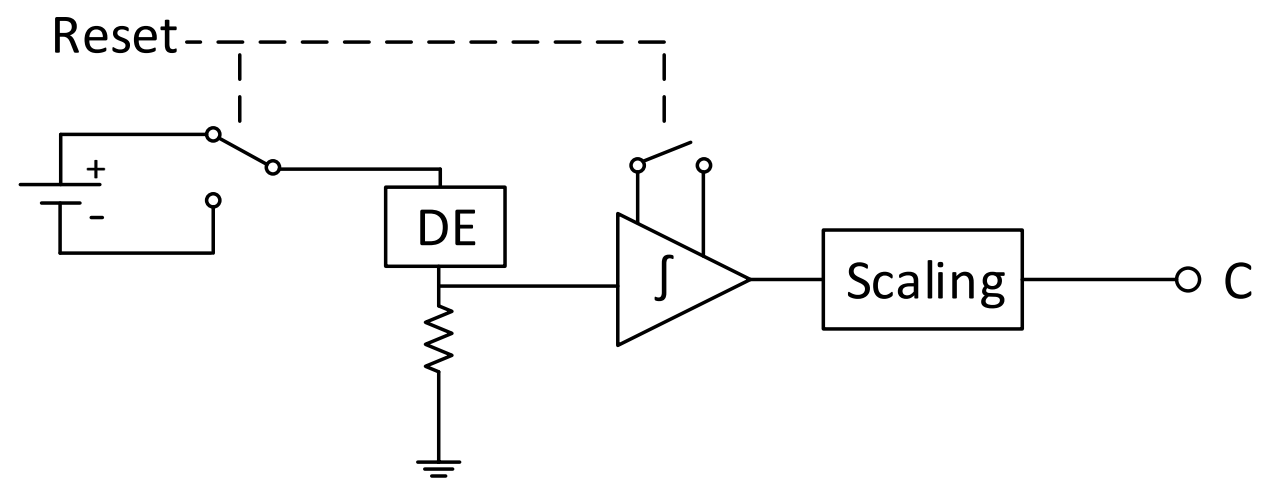

Figure 8. An analogue implementation of the charge tracking capacitive sensing circuit.

\section{DISCUSSION}

The charge tracking capacitive sensing method can be implemented with simple electronics entirely in hardware. The ability to simultaneously measure many sensors enables new applications such as measuring hand motion. Sensors can be integrated into a wearable glove or even applied directly onto the hand (Figure 9).

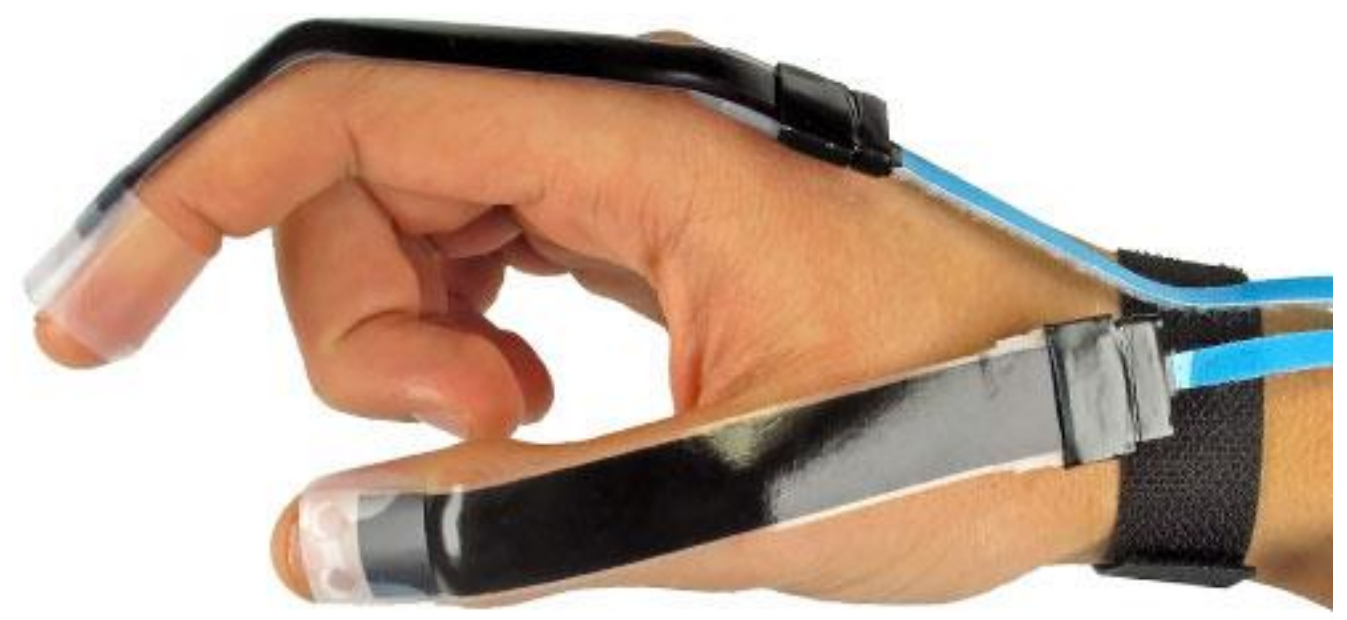

Figure 9. DE sensors applied directly onto the hand to measure motion and behaviour. (Image courtesy of StretchSense Ltd www.stretchsense.com).

A common DC voltage can be applied to all the sensors. Parallel integrators and scaling multipliers can be used to convert each sensor's capacitance to an analogue voltage (Figure 10). This creates a simple interface to any AI system. Alternatively, a multiplexing design can be used to further reduce component count ${ }^{14}$. The hardware design of charge tracking is compact and portable, with no requirement on external processors. 


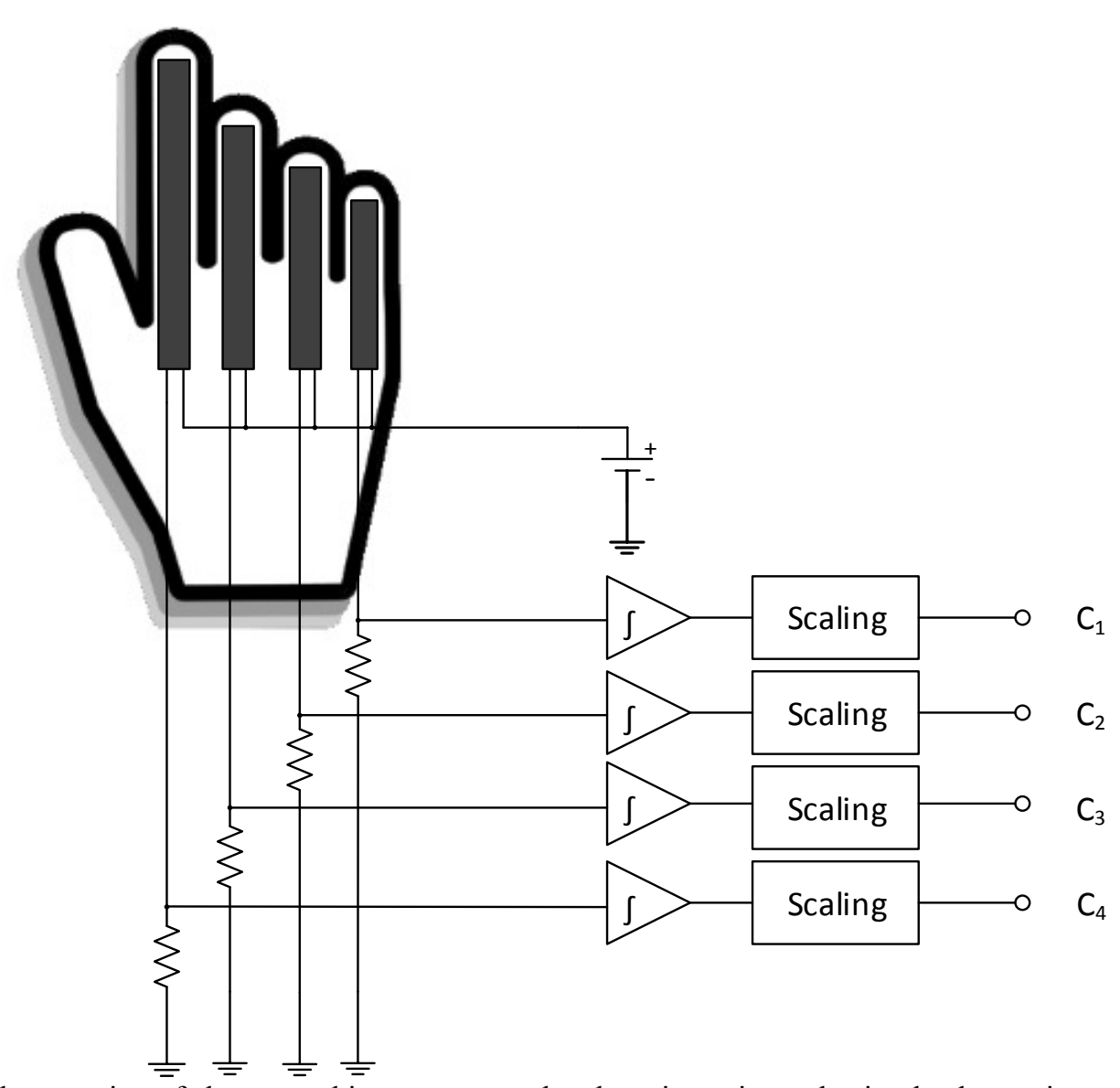

Figure 10. Implementation of charge tracking to measure hand motion using only simple electronics.

\section{CONCLUSION}

Artificial intelligence in computers and personal devices is playing a greater part in our lives. To improve their intelligence, our hand motion can be used to train AI to better understand our needs. This allows them to perform tasks customized to our behaviour. A fast, efficient and scalable capacitive sensing method termed "charge tracking" is presented for wearable dielectric elastomer strain sensors. The method uses a DC sensing voltage and continuously tracks charge movement to determine the capacitance. This method allows fast, high resolution feedback to be obtained, bringing us closer to smarter human-computer interactions.

\section{ACKNOWLEDGEMENTS}

The authors would like to thank The University of Auckland Doctoral Scholarship and StretchSense Ltd for support carrying out this work.

\section{REFERENCES}

[1] Pavlovic, V., Sharma, R., and Huang, T., "Visual interpretation of hand gestures for human-computer interaction: A review," IEEE Transactions on Pattern Analysis and Machine Intelligence 19(7), 677-695 (1997).

[2] ElKoura, G., and Singh, K., "Handrix: animating the human hand," in ACM SIGGRAPH/EG Symp. Comput. Animat.(2003). 
[3] Anderson, I.A., Gisby, T.A., McKay, T.G., O’Brien, B.M., and Calius, E.P., "Multi-functional dielectric elastomer artificial muscles for soft and smart machines," Journal of Applied Physics 112(4), 041101 (2012).

[4] Madden, J.D.W., Vandesteeg, N. a., Anquetil, P. a., Madden, P.G. a., Takshi, a., Pytel, R.Z., Lafontaine, S.R., Wieringa, P. a., and Hunter, I.W., "Artificial Muscle Technology: Physical Principles and Naval Prospects," IEEE Journal of Oceanic Engineering 29(3), 706-728 (2004).

[5] Keplinger, C., Kaltenbrunner, M., Arnold, N., and Bauer, S., "Capacitive extensometry for transient strain analysis of dielectric elastomer actuators," Applied Physics Letters 92(19), 192903 (2008).

[6] Buchberger, G., Mayrhofer, B., Jakoby, B., Hilber, W., and Bauer, S., "Dynamic capacitive extensometry setup for in-situ monitoring of dielectric elastomer actuators," Instrumentation and Measurement Technology Conference (I2MTC), 2012 IEEE International (2012).

[7] O'Brien, B., Thode, J., Calius, E.P.E., Haemmerle, E., Xie, S., and Anderson, I., "Integrated extension sensor based on resistance and voltage measurement for a dielectric elastomer," Proc. SPIE 6524, 652415-652415-11 (2007).

[8] Haus, H., Matysek, M., Mößinger, H., and Schlaak, H.F., "Modelling and characterization of dielectric elastomer stack actuators," Smart Materials and Structures 22(10), 104009 (2013).

[9] Jung, K., Kim, K.J., and Choi, H.R., “A self-sensing dielectric elastomer actuator," Sensors and Actuators A: Physical 143(2), 343-351 (2008).

[10] Gisby, T.A., O’Brien, B.M., and Anderson, I.A., "Self sensing feedback for dielectric elastomer actuators," Applied Physics Letters 102(19), 193703 (2013).

[11] Carpi, F., Chiarelli, P., Mazzoldi, A., and De Rossi, D., "Electromechanical characterisation of dielectric elastomer planar actuators: comparative evaluation of different electrode materials and different counterloads," Sensors and Actuators A: Physical 107(1), 85-95 (2003).

[12] Gisby, T.A., “Smart Artificial Muscles," University of Auckland (2011).

[13] Matysek, M., Haus, H., Moessinger, H., Brokken, D., Lotz, P., and Schlaak, H.F., "Combined Driving and Sensing Circuitry for Dielectric Elastomer Actuators in mobile applications," Proc. SPIE 7976 7976, 797612-797612-11 (2011).

[14] Xu, D., Gisby, T.A., Xie, S., and Anderson, I.A., "Scalable sensing electronics towards a motion capture suit," in Proc. SPIE 8687, 86872L (2013). 